Revue de philosophie et de sciences humaines

45-46 | 2021

Art et politique, une approche critique

\title{
Art et politique : destins croisés
}

\section{Olivier Long}

\section{OpenEdition}

Journals

Édition électronique

URL : http://journals.openedition.org/leportique/3760

DOI : $10.4000 /$ leportique.3760

ISSN : $1777-5280$

\section{Éditeur}

Association "Les Amis du Portique"

\section{Édition imprimée}

Date de publication : 10 janvier 2021

Pagination : 115-129

ISBN : 978-2-916332-48-2

ISSN : 1283-8594

\section{Référence électronique}

Olivier Long, "Art et politique : destins croisés », Le Portique [En ligne], 45-46 | 2021, document 7, mis en ligne le 10 mars 2021, consulté le 25 mars 2021. URL : http://journals.openedition.org/leportique/ 3760 ; DOI : https://doi.org/10.4000/leportique.3760

Ce document a été généré automatiquement le 25 mars 2021.

Tous droits réservés 


\section{Art et politique : destins croisés}

\section{Olivier Long}

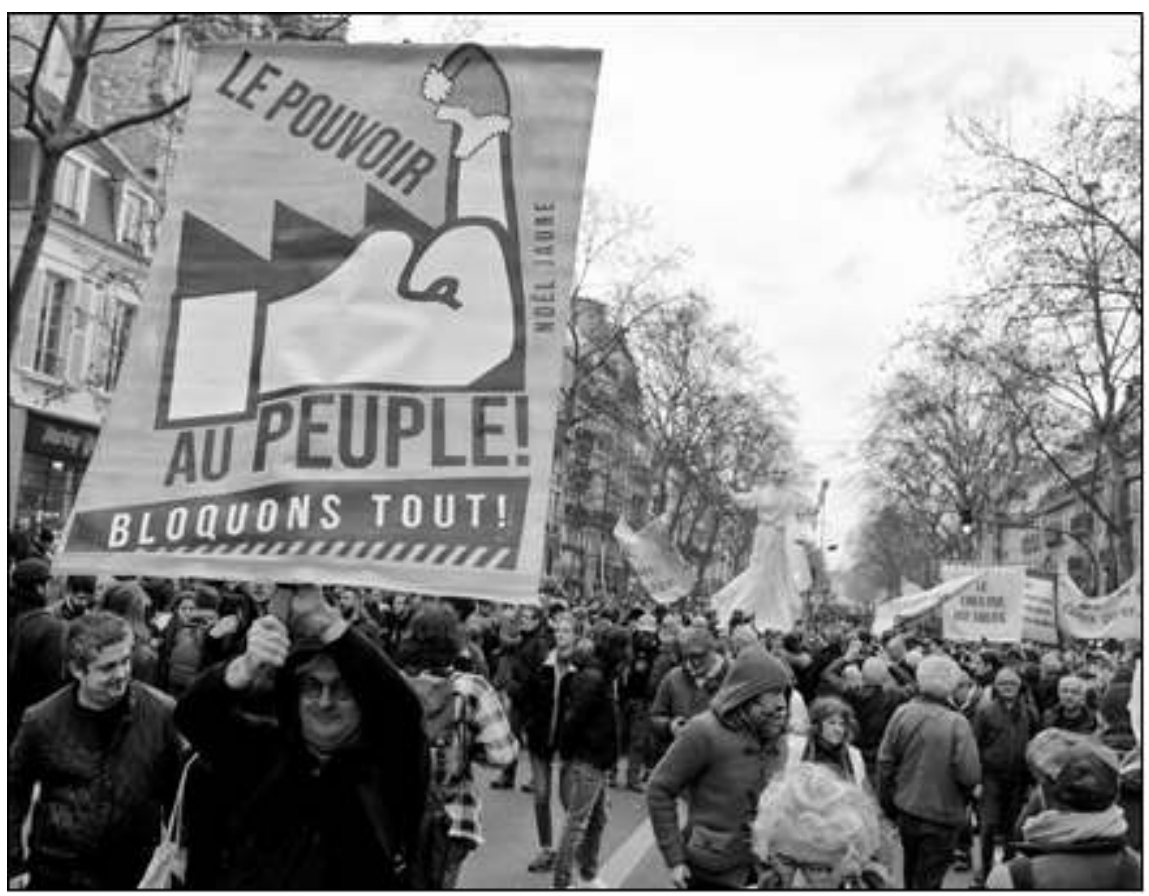

Olivier Long, Le pouvoir au peuple, affiche, acrylique sur toile, 100x140cm, 17 décembre 2019, manifestation à Paris pour les Retraites, Photo de Serge d'Ignazio.

1 Quand l'indignation passe les mots, l'invisibilisation des dominés néantise toute revendication verbalisable. Le dire échappe à tout échange comme à toute stratégie de communication, le non-dit se déploie dès lors hors de l'employabilité ordinaire du langage. Des pratiques de rue, proches de l'art, manifestent alors la face cachée de toute parole. 


\section{Le « grand débat » : une révolution?}

2 Sur les forums de discussion on débat, à la télévision on débat, avec les chaines d'information le grand débat s'écoule jour et nuit au rythme d'une lente perfusion. La globalisation du débat semble un effet de la numérisation du monde. Dès l'origine l'internet a été inventé pour que des chercheurs puissent exposer leurs thèses et débattre de leurs résultats. En se démocratisant cet outil de digitalisation et de calcul des existences promettait un espace de liberté infini fondé sur une délibération totale : une " cyberdémocratie » disait-on. Selon ses promesses la technologisation de "l'agir communicationnel » (théorisé par Habermas dans les années 1980) devait avoir pour conséquence inédite le développement d'une intelligence collective ${ }^{1}$ liée à l'échange planétaire d'arguments.

3 Trente ans plus tard, la principale convivialité qui fonde les «démocraties» autoritaires reste le débat. Partout où s'impose l'évidence d'une guerre sociale, ça débat. Le débat est l'alibi des pseudo-démocraties.

4 Vous débattez avec votre banquier, vous débattez avec votre médecin (la discussion a été préparée sur Doctissimo), vous débattez avec votre patron. "Avez-vous des questions ? » : c'est la phrase qui clôt le rituel de n'importe quel entretien-débat d'embauche. Un entretien de licenciement et vous voilà convoqué pour une passionnante discussion : à vous d'alimenter le débat afin d'organiser l'interminable procession qui consacre votre enterrement social. Le débat est devenu la fondation de toute sociabilité comme de toute société : il consacre du sceau de la collectivité n'importe quel moment d'existence.

5 Comme toute sociabilité c'est un rite. Qu'attendent les gens du rite ? Une sorte d'épiphanie sociale: le rite relie, le rite transforme, le rite consacre les grandes occasions. On se souvient du débat télévisé qui précipita Emmanuel Macron au pouvoir: ce débat avait tourné aux jeux du cirque, ces rituels de triomphe qui consacraient tout candidat à l'imperium. Emmanuel Macron contre Marine Le Pen était un combat avec estocade, mise à mort finale et intronisation du vainqueur. " La démocratie, c'est le débat, et le contraire de la violence » : cette antienne est répétée en introduction de n'importe quelle grande liturgie médiatique.

6 Une émeute (je pense ici aux premières journées d'émeutes parisiennes de l'hiver 2018 où Paris avait été mis "à feu et à sang " selon l'expression d'un membre du gouvernement) ? On y répond par un " grand débat ": " venez discuter plutôt qu'occuper les rues et envahir les ronds-points ". Le président-philosophe (ou journaliste ?) se fait l'éditorialiste de sa propre politique : voilà qu'il débat avec luimême et qu'on assiste à ce long colloque qui montre l'émergence publique du grand fleuve de ses pensées. Le monologue décevra les intellectuels de cour qui ont un moment pensé que le président les avait réunis pour profiter de leurs sages conseils. Le grand débat macronien mime un grand débat plus ancien : celui institué par la réunion des États généraux de l'hiver 1789. Si ce débat avait rapidement dérapé lors du serment $\mathrm{du}$ jeu de paume, n'oublions pas qu'il avait à l'origine une cause identique : une crise économique et des émeutes récurrentes depuis plusieurs années. Le grand débat de 1789 avait pour but de résoudre une crise financière afin d'apporter " remède aux grands maux de l'État $»^{2}$ et assurer " la félicité publique ». Il n'en fut rien, si bien qu'on peut se demander ce qui relie le débat comme rituel démocratique et la notion de gouvernementalité dans les sociétés modernes. 
7 Dans d'autres sociétés les bals de villages, les cérémonies d'initiation, les scarifications, les jeux périlleux, les déformations des lèvres et des oreilles, l'excision, la chasse aux fauves, les rites d'aspersion, les libations, l'ingestion d'alcool, de psychotropes, les enterrements par crémation, les baptêmes par immersion, les processions, l'enterrement de la vie de jeune homme ou de jeune fille ceci et mille autres choses fondent le lien social. La question demeure de savoir pourquoi le débat est devenu la forme rituelle et magique des sociétés médiatiques autoritaires.

8 Si le grand débat de 1789 a pu démontrer les contradictions d'une société pétrie d'intérêts divergents, le grand-débat actuel est un rite d'inhibition de l'agressivité sociale. En tant que rite, il structure la hiérarchisation des dominations plutôt qu'il ne la questionne. Le débat démocratique actuel n'aurait-il pour but, comme rituel politique, qu'une poursuite des discriminations sociales ? Ce qui s'y négocie, n'est-ce pas simplement comme dans tout rite l'accaparement d'avantages matériels, alimentaires, sexuels, territoriaux ou sociaux?

\section{Le grand débat, roman familial du candidat Macron}

On ne prend pas soin de lire la littérature présidentielle, mais il est significatif que le chapitre inaugural de l'ouvrage d'Emmanuel Macron titré Révolution commence par un récit de genèse de la parole usant de toutes les ficelles du roman familial. On est surpris que ce livre programmatique commence par un vibrant hommage du candidat à la présidence par un hommage à... sa grand-mère. Une grand-mère avec qui il débat.

De grand-mère en petit-fils, dans la famille Macron, on débat, et c'est peut-être pour cela que l'ouvrage s'intitule précisément Révolution. Cette grand-mère, enseignante, ayant gravit l'échelle sociale à la force du poignet, aurait tout appris au jeune président de l'ascension sociale en débattant avec lui. Le futur président décrit la relation avec cette grand-mère comme un face à face exclusif, dans lequel les ascendants directs (père et mère médecins) ne sont évoqués que très rapidement. Pour ce qui est des autres membres de la fratrie (frères et sœurs) ils suivent la voie professionnelle des parents, faisant corps avec l'anonymat parental. Le petit Emmanuel (dont le nom consacre l'élection divine, il signifie " Dieu est avec nous ») se distingue en suivant quant à lui une trace toute différente : celle d'une enseignante sortie de sa condition paysanne à la force du poignet. Telle Énée fuyant Troie en flamme, cette grand-mère quitte ses montagnes, elle porte sur le dos sa propre mère, l'arrière-grand-mère présidentielle. Il est précisé que cette dernière était une « femme battue » et qu'elle ne dut son salut qu'au parcours héroïque de sa jeune fille. Devenue fonctionnaire, cette situation lui permit une mutation décisive pour échapper à la violence du grand-père. Le parcours du jeune Emmanuel semble la répétition de cette scène primitive : le colloque exclusif et perpétuel avec la grand-mère délivre de la malédiction d'être battu (à la présidentielle ?), afin de devenir l'élu des cœurs et de conquérir tous les suffrages.

11 Comment échapper à la violence primitive ? Dans un devenir de président-philosophe au moyen d'incessants dialogues entretenus avec sa grand-mère-nourricière. Brigitte Trogneux, enseignante de théâtre à laquelle le jeune Emmanuel - devenu lycéen donne la réplique, redouble la figure ancestrale et dialogique d'une grand-mère qui hante depuis toujours le candidat Macron. Le chapitre premier de Révolution décrit comment la relation avec sa future femme poursuit par des discussions passionnantes le grand-ébat commencé avec la grand-mère. Grand-mère, grand débat, grand ébat font 
système. Naître à la réussite, vaincre, c'est se battre en débattant. D'où la figure d'un président- philosophe gagnant parce que débattant, donc jamais battu ni abattu par des obstacles qui sont autant d'arguments à affronter pour réussir à grandir comme être humain, puisqu'être humain c'est d'abord s'élever dans la hiérarchie sociale. Rappelons que les romans familiaux ont pour objectif de surmonter la honte d'être mal-né, mais fait remarquable dans ce cas - la honte est ici surmontée par le grand débat. C'est la raison pour laquelle on a pu noter que " Macron parle, parle, parle " .

Derrière ces histoires de gynécée, d'exclusivité maternelle et d'enfermement dans un univers cloîtré se cache pourtant une parole paternelle. Le candidat à la présidence poursuit l'hommage inaugural à la grand-mère par le vibrant éloge d'une figure professorale : Paul Ricœur. De manière surprenante, Emmanuel Macron précise immédiatement qu'il n'a lu aucun de ses livres, pour ajouter très rapidement qu'il eut avec Paul Ricœur de passionnantes discussions. C'est à nouveau l'oralité qui constitue Ricœur en mentor et en miroir de la grand-mère. Quand ce pédagogue prend le relais de l'univers familial et familier, il prend la figure d'un père d'adoption de la même manière que la grand-mère avait pu devenir une mère de substitution. On note qu'à nouveau la transmission générationnelle n'est pas affaire de corps, fusse le corps d'un texte écrit, mais de parole, il s'agit d'une transmission strictement mentale qui fait du rapport corporel l'objet d'un déni systématique, puisque la violence des ancêtres a démontré que par le corps ne passent que les coups d'une violence masculine qu'il faut fuir.

Quiconque essaie de comprendre le grand débat macronien, la surdité et les distorsions de langage qui l'accompagnent est inéluctablement renvoyé à ce roman familial qui institue comme projet de rénovation totale de la vie politique cette scène primitive. On est inévitablement renvoyé aux thèses de Marthe Robert. Selon Marthe Robert, le roman familial comme genre littéraire ne relève pas du roman, mais de la magie. C'est " une parole rituelle qui doit être exprimée pour que le romancier parvienne à se libérer d'un fantasme trop astreignant $»^{4}$.

Considérons Révolution comme un acte littéraire, liturgique, magique et programmatique, un acte rituel comme le seront les grand-messes macroniennes : les conseils des ministres organisés à Versailles, l'entrée solennelle par la cour du Louvre (ancien palais des Tuileries) du président nouvellement élu, les rencontres avec les « intellectuels ». Depuis le concile de Trente, on multiplie les rituels là où la présence réelle du corps manque, et surtout là où la légitimité temporelle fait défaut. Pour mieux incarner la puissance, il fallait aux rois d'antan un double corps, corps terrestre et corps céleste, qui leur permettait de délibérer « en leur conseil », comme le disent les anglais, c'est-à-dire au moyen d'un parlement. Ce lieu de débat redoublait la puissance mystique du corps royal. Mais que reste-t-il de ce double corps quand n'existe plus de corps électoral sur lequel s'appuyer (rappelons qu'Emmanuel Macron a été élu par une minorité du corps électoral) ? Quand un pays entier se retrouve soumis à la puissance d'un fantasme, comment s'étonner qu'il ne reste qu'une indignation qui passe les mots pour s'opposer à la toute-puissance d'une profération sans corps?

\section{En dialogue avec Ricœur}

15 C'est dans les thèses de Paul Ricœur que se situent les apories de la posture présidentielle et de l'insupportable dialogue avec lui-même que le long monologue macronien met en scène depuis plusieurs années. Ce qui fonde ces apories renvoie 
selon nous au paradigme humaniste d'un monologue dialogique fondé sur l'imitation littéraire. Un peu d'archéologie des discours s'avère nécessaire pour comprendre la faiblesse de cette position qui fait de la politique une affaire d'énonciation individuelle du soi.

Paul Ricœur ${ }^{5}$ enseignait dans les années 1990, suite à un croisement de ses travaux sur la théorie américaine de l'action et des théoriciens russes de la narration, que l'assertivité de soi, l'affirmation d'un rapport à soi comme autre qu'il nommait « ipséité " (Soi-même comme un autre est le titre d'un de ses ouvrages phare), n'était qu'un long travail de reconfiguration dialectique du même et de l'autre opéré à la faveur de la mise en intrigue des grands récits de la grande littérature. "Ricour distingue en effet, [...] la mêmeté de l'ipséité. La mêmeté évoque le caractère du sujet dans ce qu'il a d'immuable, à la manière de ses empreintes digitales, alors que l'ipséité renvoie à la temporalité. ${ }^{6} \mathrm{Ceci}$ signifie chez Ricœur que, pour éviter le piège de l'illusion d'une auto-saisie de soi cartésienne (relation sujet-objet), ne reste au sujet que l'altérisation de soi par le long dialogue avec des œuvres qui le précèdent.

Cette problématisation de soi comme récit dialogique, Paul Ricœur l'avait envisagée dès Temps et Récit ${ }^{7}$ dans les années 1980. Inspiré par Propp, il a travaillé à l'idée d'une genèse mutuelle du caractère de la personne portée par le fil d'une mise en intrigue. Le récit opère selon lui une transformation de l'agir et du sentir du lecteur en reconfigurant ses possibilités d'identification et par conséquent d'action. Cette attestation d'un « je » passé au fil de l'intrigue est le processus d'identification nécessaire qui conditionne selon lui la transformation d'une identité immédiate pour qu'elle se ressaisisse comme ipséité. Et c'est ce dialogue avec un texte qui opère la transmutation d'un soi souffrant en sujet d'action éthique. L'ipséité ne se transfigure en altérité qu'altérée par le débat avec les œuvres. Ce processus permet à la première personne, au " je » de s'attester comme promesse et comme souci de l'autre. C'est à cette condition que « je » peut s'énoncer performativement pour dire : « ici je me tiens ». C'est donc la " compréhension du texte » comme "compréhension de soi » qui conditionne une attestation épistémologique de " je » dans un jeu de différence et d'identité au fil des textes.

Cet « ici je me tiens " de Ricœur, le jeune Macron l'a intégré comme construction dialogique de soi. À n'en pas douter cette attestation solitaire de soi est bien à l'œuvre quand le président Macron comprend son action individuelle et solipsiste comme souci et saisie de soi, d'où l'aspect littéraire, très écrit de n'importe quelle intervention présidentielle. Le dialogue avec les œuvres est dialogue avec lui-même dans une délibération intérieure qui se pense dialogue avec d'autres. Qu'est-ce que la politique dans ce cadre ? C'est la projection de ce colloque sur la scène médiatique pour effectuer la démonstration de la puissance d'agir d'un pouvoir politique impuissant sur la misère réelle des gens.

Ainsi, si les réformes passent mal, c'est parce qu'elles n'ont pas été assez expliquées. Car dans ce cadre, expliquer c'est comprendre. L'herméneute ricœurien opère dans un cercle qui consiste à comprendre pour s'expliquer et s'expliquer pour comprendre selon un vieux paradigme heideggérien ${ }^{8}:$ le " cercle herméneutique ». C'est la clôture de ce cercle qui fait du dialogue avec les textes une activité d'altérisation au contact des œuvres. Les grands récits sont le miroir d'une mêmeté brisée, ils garantissent l'infinitude d'un désir d'altérité enfermé dans la sacralité des textes. 

est-il un rituel suffisant pour intégrer la corporéité d'un texte ? La disparition d'une intégration corporelle et rituelle du texte est un problème théologique non élucidé dans le protestantisme intellectualiste de Ricœur. Ce problème est lié à l'individualisation des pratiques de lecture dans le protestantisme réformé. Sans revenir à la sola scriptura des premiers réformateurs, il suffit de comparer les pratiques d'étude collectives dans le judaïsme talmudique et l'herméneutique de Rudolf Bultmann - dont Ricœur est un grand lecteur -, pour comprendre l'aspect souvent solipsiste de l'herméneutique ricœurienne. Commentant Bultmann, Ricœur avance :

"L'idée même d'annonce, de proclamation, de kérygme ${ }^{9}$, suppose, si j'ose dire, une initiative $d u$ sens, une venue à nous du sens, qui fait de la parole, un vis-à-vis de la décision existentielle. ${ }^{10}$

Pour Ricœur, c'est par cet acte d'écoute et de lecture que « le sens fait face au lecteur ${ }^{11}$. Or ce qui fait face dans le face à face (Panim el panim) biblique, ce n'est pas un texte mais un autre, c'est-à-dire un événement inédit, inouï et non-interprétable qui suspend le sens de tout texte. Le Panim el panim est à comprendre en dehors de tout personnalisme. L'autre en face, c'est déjà du collectif et plus du personnel.

Cette problématisation personnaliste de l'interprétation des textes et de l'autre comme texte, au travers de pratiques de lectures spécifiques n'est pas sans conséquence sur la posture solipsiste de l'action du futur président Macron.

\section{Rome et la Révolution : la représentation politique comme débat}

Le problème chez Ricœur est que son rapport au texte est beaucoup moins lié au monde biblique comme il le pense (Le Talmud est un espace d'incorporation très ritualisé), qu'il ne cache sa dette envers des pratiques de lecture typiquement humanistes.

Ricœur se réfère à la Rhétorique d'Aristote, à la philosophie américaine de l'action mais c'est moins à ces références qu'aux pratiques sociales de la rhétorique romaine et de ses implications éthiques qu'il fait référence quand il formalise sa philosophie de l'interprétation comme philosophie de l'action : sa philosophie est de part en part liée à un paradigme classique.

25 N'oublions pas que le modèle du grand débat lors de la Révolution française fut une question de juste représentation des trois états de la société de l'époque : noblesse, clergé et le tiers-État. Toute la question était de savoir quelle devait être la proportion de représentants de chaque corps dans une royauté en train de basculer dans une monarchie constitutionnelle. C'est en étudiant l'idéologie romaine de l'art oratoire et les pratiques d'assemblées du forum romain, qu'on peut comprendre comment ces pratiques de débat constituèrent un modèle de gouvernementalité pour l'époque révolutionnaire. C'est dans des pratiques sociales de l'Empire romain (et surtout dans leurs ambiguïtés) que l'utopie émancipatrice de la grande Révolution a puisé pour instituer une représentation du corps social.

Nous voici rendus aux pratiques de débat sur le forum romain et aux théorisations de l'art oratoire antique. "Combien de charme la parole ajoute à la beauté morale ", affirme Plutarque dans sa Vie de Cicéron ${ }^{12}$. Dans l'Institution oratoire de Quintilien, un des principaux théoriciens romains de l'art oratoire, le jeune rhéteur ne devient Vir bonus, " homme de bien » qu'en élargissant sa culture littéraire. Toutefois chez Quintilien ce projet de formation oratoire et citoyenne mute spectaculairement en projet 
d'éducation totale. La machine à fabriquer des orateurs, des " hommes bons ", des " types bien » dès le berceau devient un art de vivre à part entière. Cet idéal définira l'art de vivre dialogique de l'humanisme lettré à partir de la Renaissance. C'est ainsi que dans une optique romaine, l'excellence oratoire a peu à peu coïncidé avec l'excellence humaine, elle a fini par se confondre avec ce que les philosophes grecs appelaient « la vie bonne ", la vie philosophique telle que la définissait les Grecs. Ce qui pose ici problème, c'est que concrètement, à Rome, la fonction d'orateur était liée au système de patronage, de redistribution et d'échange qui commandait les échanges de la société romaine. L'idéologie du "bon orateur " que véhiculent les traités de rhétorique romaine n'est que le strict décalque d'un rapport de domination sociale ${ }^{13}$. Ce qui a pour conséquence directe de placer l'échange d'idées dans une situation de dissymétrie. La seule égalité qui peut en résulter n'est pas celle des arguments, mais celle d'une solidarité de dominants qui ont seuls accès à la parole.

C'est dans ce cadre que se développe une politique de l'amitié. Au travers d'une culture de lectures partagées, le jeune orateur découvre son appartenance à une communauté sociale de dialogue. Cette découverte fait de l'autre un ami lecteur avec lequel il débat au forum. Au travers d'un jeu complexe d'alliance clientélaire et de patronage complexe, à Rome, le " sage éloquent " se découvre dans l'acte de lecture une intensification relationnelle qui est puissance de domination sociale. C'est cette culture du débat épistolaire transférée dans les cercles humanistes qu'on appellera « république des lettres » et dont on fera l'origine mythique de la Révolution française. Mais cette institution de la politique comme objet de débat trouve d'abord ses repères dans les hiérarchies discriminantes de la société romaine.

La lecture comme dialogue avec soi n'est pas un partage cosmopolite, c'est un art qui, $\mathrm{du}$ forum à la rhétorique du grand siècle, fonctionne comme puissance de discrimination sociale. L'amitié des gens de bien comme acte dialogique, ce que Cicéron nomme humanitas, désigne ce processus au fondement de l'humanisme. L'amitié cicéronienne est un cosmopolitisme de lettrés dominants qui font d'un canon littéraire la clef d'un " cosmopolitisme » de soi-même. C'est ainsi qu'en Occident, sous prétexte de produire dans un échange de paroles une communauté d'interprètes, l'étude des lettres a été comprise en vue de performances oratoires publiques, dans un but de transmission d'un rapport de domination politique. De Gaulle, Pompidou et Mitterrand se sont tous voulus de grands lettrés ; Alain Juppé, ce " normalien sachant écrire " selon les mots de Chirac, ne fait pas figure d'exception. Il est le mentor du premier ministre Édouard Philippe. L'idéologie postmoderne et un peu has been du grand débat macronien, est en fait prédéterminée par une culture des maîtres brutale : celle de la romanité. Ce sont ces ambiguïtés héritées de la Révolution française et de son modèle antique que la tradition des grands débats d'aujourd'hui tente de réactiver au profit d'un nouvel empire rhétorique qui consacre la politique comme collusion de l'économie et des médias.

\section{Gilets jaunes face à l'anéantissement : « Nous, on est là »}

29 " On est là, on est là, ... nous on est là... ", tel est le slogan des gilets jaunes dès les derniers mois de l'hiver 2018. Autrement dit : on ne vient pas discuter, on est simplement là. Être là c'est être présent sans procuration ni représentation, sans délégation de parole ni de pouvoir. Face à qui vous oublie ou vous écrase, reste ce qui demeure : de 
l'entêtement d'une présence, loin des grands discours, naît une forme très particulière de perception d'une néantisation de soi.

Contrairement à la fausse égalité que suppose l'institution d'un grand débat, les émeutes de l'hiver 2018 n'échangèrent que peu d'arguments ni ne présentèrent aucune preuve. On le leur reprocha en faisant semblant de croire que puisqu'elles ne portaient pas d'arguments, il s'agissait d'une revendication nécessairement confuse. Dans ces événements sourds pourtant l'expression d'une présence brute (" on est là »), et cette présence est adressée à qui fait face. Tout ceci parce qu'il y a quelque chose de demeuré inexprimé, inexprimable, inécoutable sans être inaudible dans ce qui advient des vies. Ce cri d'une indignation qui passe les mots a quelque chose à voir avec l'irreprésentable puisqu'il naît de l'invisibilisation qui produit l'invisibilité des " gens de rien ». Son lieu naturel c'est la sidération c'est-à-dire le silence qu'engendre la violence invisible des dominants. C'est une parole muette qui naît d'une négation fondamentale, elle porte alors le déni des dominants là où ils ne s'y attendent pas, à la puissance deux. Cette négativité n'est pas l'antithèse d'une position adverse, mais l'écho d'une autre négation qu'elle traverse pour s'augmenter en intensité. C'est négation contre négation et non pas négation contre position. Néant ou nihilisme ? À la rigueur sur-néant, puissance de surnéantisation quand le tort fait par l'autre provoque l'inouï d'une expression qui s'impose comme surprésence face à ce qui transcende le dire. Ici la parole n'est plus production de significations ayant vocation à être communiquées mais événement pur. Et si l'on continue de parler c'est pour tenter de dire, comme un manifestant crie son indignation : ce qui passe les mots quand, face à l'anéantissement, s'effondre le réseau des illusions qui constituait jour après jour le tissu des existences et des mots qui les portent.

Que disaient les Enragés de 1789 ? " Notre patience est à bout ", ce qui signifie que la Révolution est d'abord possible puisqu'elle a lieu et que ce lieu naît du néant, c'est-àdire de la négation de toute position possible. C'est le déni du déni de l'autre qui engendre un rapport d'expression. Ce qui porte ici le cri c'est l'impossibilité de pouvoir faire autrement. Quelque chose doit être dit qui ne peut souffrir de ne pas l'être. Si bien qu'on peut se demander quel est ce noyau d'être, de présence irréductible qui ne se laisse réduire par aucun débat et qui constitue pourtant la présence d'un «nous » sinon d'un « on »? La communauté invisible des invisibilisés est ce point à partir duquel ce " nous » se tient là, par nécessité, comme présence immédiate au présent, et qui déborde de surprésence. Durant l'hiver 2018 et jusqu'au mois de novembre 2019, le rassemblement de ces simplicités eut pour lieu les rond points.

Le discours, le dialogue, le grand débat, ne sont que des ruses de prêtres, afin de manipuler cette puissance de négativité pour la rendre accessible à leur profit. Les grands discours, les sermons, les dialogues et controverses théologiques sont des outils de manipulation afin d'émousser, de désamorcer le désordre fondamental qu'introduit dans l'ordre civil les puissances de néantisation produites par l'invisibilisation des dominés.

C'est ici qu'à mon sens peinture et politique partagent quelque chose de commun, un croisement de destins, sans que cela ne constitue ou ne déclare une quelconque obligation ancillaire. L'art comme subversion (ce qui est un pléonasme puisque le devenir-artiste est intrinsèquement lié à l'incapacité à se soumettre comme le démontre de nombreux parcours de peintres) est le contraire de l'État comme institution, son lieu naturel c'est l'impossibilité d'être. 

qui détruit ce qui empêche de vivre.
ce déni est une force qui fait tenir.



Olivier Long, MACRON/ONCRAM, Affiche, 2019 n'est pas sa face inverse mais son dehors. dans leur négation $»^{16}$.

L'art est comme le cortège de Dionysos qui se promène devant les athéniens sidérés : il sort directement des enfers pour aller dire aux vivants leur peu d'existence. Cette présence qui vient des morts, aucune institution ne peut la convoquer, aucun appel ne peut la domestiquer ni la conjurer puisqu'elle est l'envers de la réalité. Dionysos nous apprend que la réalité est une pièce, mais qu'elle n'a qu'une face. Dès lors son envers la peinture c'est l'humain devant sa débâcle. « En peignant, je repousse ce monde qui empêche la vie et où on risque constamment d'être écrasé [...]. L'artiste vit un secret qu'il lui faut manifester $»^{14}$, faisait remarquer le peintre Bram Van de Velde. Ces mots permettent de saisir la mesure de cette zone d'invisibilité qui provoque l'acte de peindre. C'est pourquoi il est inutile de peindre quand il n'y a pas urgence, peindre se fait toujours plus ou moins dans la panique. Ce qui différencie l'art du débat c'est que l'art « ne cherche pas à convaincre, à prouver quoi que ce soit ». Cézanne ne contredit pas Michel-Ange, c'est pourquoi ils n'argumentent pas ensemble, ne discutent des goûts et des couleurs que les critiques ou les marchands. Pour les autres, chaque chemin est une approche d'une zone d'inaccessibilité d'où sort l'art comme révolte.

Barthes disait : " Il est impossible de représenter le politique. Contrairement à la croyance invétérée de tous les arts socialistes. Là où commence la politique, là cesse l'imitation. $»^{15}$ En 1789 comme en 2018, c'est du surgissement qu'émerge l'émeute moins que d'une revendication argumentée. C'est la raison pour laquelle, comme le fait remarquer Kropotkine dans La Grande Révolution, " si les idées du peuple étaient confuses d'un point positif, elles étaient au contraire très nettes sous certains rapports,

Comme la peinture détruit ce qui empêche de voir, le soulèvement est la part de mort qui détruit ce qui empêche de vivre. On se soulève comme on peint : face à l'écrasement

-------------------------- 


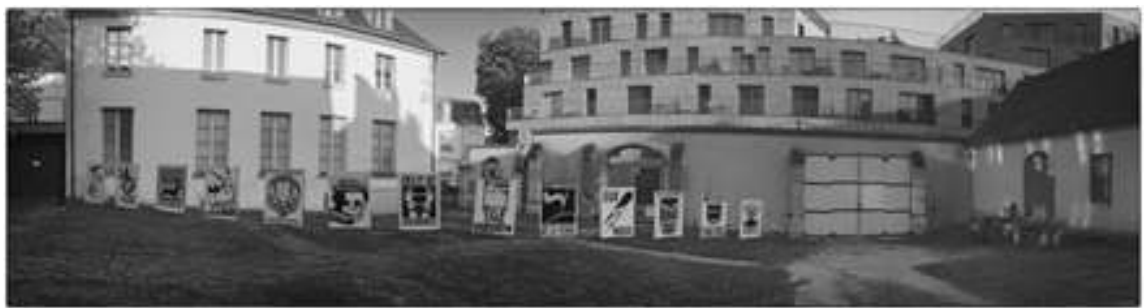

Olivier Long, « Mes amours jaunes » installation pour l'exposition « Enfermement », musée d'art et d'histoire de Saint-Denis. @ Jean-Michel Fickinger

\section{NOTES}

1. Pierre Levy, L'Intelligence collective. Pour une anthropologie du cyberespace, Paris, La Découverte, 1994.

2. Discours de Louis XVI, donné à Versailles, le 24 janvier 1789.

3. Frédéric Lordon, « Le moment Potemkine », La Pompe à phynance, Blog du monde diplomatique, 13 décembre 2019.

4. Jean-Pierre Goldstein, « Marthe Robert, Roman des origines et origines du roman ", in Études Littéraires, 1973, p. 120.

5. Paul Ricœur, Soi-même comme un autre, "Sixième étude ", éditions du Seuil, Paris, 1990, pp. 167-198.

6. Voir François Dosse, «L'importance de l'œuvre de Paul Ricœur », dans le Bulletin de la Société de l'Histoire du Protestantisme Français, vol.152, octobre- novembre-décembre 2006, p.663.

7. Paul Ricœur, Temps et Récit II, Paris, éditions du seuil, 1984, p. 60.

8. Martin Heidegger, Etre et temps, trad. par R. Boehm et A. de Waelhens, éditions Gallimard, Paris, 1964, p. 190.

9. Le "kérygme " est le noyau historique de la foi primitive des premières communautés chrétiennes. La Formgeschichte (" la critique des formes ») du théologien et exégète Rudolf Bultmann tente de le mettre à jour afin de renouveler la pratique et l'intelligence de la foi protestante dans une visée post-critique.

10. Paul Ricœur, "Préface à Bultmann ", in Le conflit des interprétations, Essais d'herméneutique, Paris, éditions du Seuil, 1969, p. 390.

11. Paul Ricœur, ibidem.

12. Plutarque, Vita Ciceronis, $13,867$.

13. Voir à ce sujet : Alain Michel, Les rapports de la rhétorique et de la philosophie dans l'œuvre de Cicéron, éditions Peeters, Louvain, 2003, pp. 19-25 et Florence Dupont, L'orateur sans visage / Essai sur l'acteur romain et son masque, éditions P.U.F., Paris, 2000, pp. 8-11.

14. Charles Juliet, Rencontres avec Bram Van de Velde, éditions P.O.L., Paris, 2016, p. 31 et 32.

15. Roland Barthes, "Qu'est-ce qui limite la représentation ? ", in Roland Barthes par Roland Barthes, éditions du Seuil, Paris, 1975, p. 157.

16. Pierre Kropotkine, La Grande Révolution, 1789-1793, éditions Stock, Paris, 1909, p. 19. 


\section{RÉSUMÉS}

Dans ce texte, le corps est élaboré comme le lieu de rencontre d'un destin croisé entre peinture et politique, lorsque « quelque chose doit être dit qui ne peut souffrir de ne pas l'être ». Suivant la piste de ce cri de la présence, l'auteur retrace les derniers mois des luttes sociales, en faisant apparaître dans l'usage politisé du « grand débat ", une généalogie qui, traversant Paul Ricœur remonte jusqu'à la Rome antique.

In this text, the author elaborates body as an encounter of destinies between painting and politics, when « something must be said that cannot suffer from not being said ». Following the line of this cry of presence, the author traces the last months of social struggles, by revealing in the political use of the "great debate", a genealogy which, crossing over the thought of Paul Ricœur, goes back to ancient Rome.

\section{AUTEUR}

\section{OLIVIER LONG}

Olivier Long est un peintre, activiste et théoricien. Il est l'auteur de l'essai L'œuvre comme exercice spirituel (Paris, 2013) et du livre Pavés Graphiques (Paris, 2018), qui recense son travail d'affiches et ses actions militantes. Les revues en ligne LundiMatin, Pratiques Picturales et d'autres accueillent régulièrement ses chroniques et interventions politiques. Il enseigne la pratique de la peinture et « l'esthétique des peintres » à la Faculté des Arts de l'Université de Paris 1 (Panthéon-Sorbonne). Son travail a été montré dans de nombreuses institutions internationales, ainsi que dans les galeries en France et en Allemagne. 